\title{
Late adolescent girls used 4 stories to describe their smoking patterns
}

Seguire M, Chalmers KI. Late adolescent female smoking.J Adv Nurs 2000 Jun;31:1422-9.

QUESTION: What are the smoking patterns of late adolescent girls?

Design

Ethnography.

\section{Setting}

6 high schools in Winnipeg, Manitoba, Canada.

\section{Participants}

25 adolescent girls aged 18 or 19 years (mean age 18 y) who were current $(n=23)$ or former smokers $(n=2)$ and attending high school. Participants were recruited using purposive sampling techniques.

\section{Methods}

Semistructured interviews of 30-40 minutes each were done in a private room in the girls' schools. Interviews were tape recorded, transcribed verbatim, and analysed using an open coding system. Categories were formed until data saturation was reached.

\section{Main findings}

4 major categories that described the smoking patterns of the late adolescent girls emerged in the form of stories.

The start story described when and why the girls began their smoking behaviour. Smoking behaviour emerged at varying ages from early puberty to late adolescence and stemmed from a need to "fit in" socially, to portray a "cool" or "mature" image, and to rebel. Peer and family influence were also expressed as factors for starting to smoke.

The smoking story described how experimental use with cigarettes progressed to regular use, which usually meant an increase in the number of cigarettes smoked. Perceived benefits of smoking were feelings of connectedness with other smokers and a method of coping with uncomfortable feelings. Few used smoking for weight control. The symbolism of smoking differed with age: for younger girls, smoking represented sophistication and defiance, whereas for older girls, smoking had a negative connotation. Maturity and self reflection led to feelings of regret for having started smoking. All girls described the addictive effects of nicotine.

The quit story described the attempts to stop smoking. Several girls were in various stages of attempting to quit and described a start stop cycle for quitting. Road blocks to quitting included withdrawal symptoms such as cravings and edginess and the positive association of smoking with drinking alcohol. Most participants identified self motivation and the emotional support of friends and family as helpful factors in smoking cessation. Nicotine gum and the patch were not perceived as aids that they would use.
The looking to the future story referred to the hopes and fears associated with smoking. Many girls hoped to be able to stop smoking in the future and then to remain "smoke free"; however, several girls had no desire to stop smoking because it was so much a part of their identity. The girls expressed a fear of the effects of smoking during pregnancy and the effects of second hand smoke on children.

\section{Conclusions}

Late adolescent girls described 4 stories that represented their smoking patterns and processes. The start story described a purposeful beginning of smoking behaviour; the smoking story described the perceived benefits, symbolisms, and regrets of smoking; the quit story described the challenging and difficult experience of trying to stop smoking; and the looking to the future story described the girls' hopes and fears about smoking in the future.
Source of funding: no external funding

For correspondence Ms M Seguire, Faculty of Nursing, University of Manitoba, Winnipeg, Manitoba R3T 2N2 Canada.Fax +1204 4747682.

\section{COMMENTARY}

Few studies have explored the smoking and quitting patterns of adolescent girls, particularly in their later years. This study by Seguire and Chalmers is one of the few studies that identifies smoking patterns and the factors that influence and support smoking cessation among older adolescent girls.

Participants in the study were secondary school students from one city in Canada. The authors do not report on ethnic and cultural variability in the sample. Therefore, the findings may not be generalisable to adolescents from different locations or cultures, or those who are not in school.

The findings are relevant to nurses who work with adolescent girls in primary health care, public health, and school settings. The finding that the functional meaning of smoking changes with age suggests that nurses need to tailor their prevention and cessation programmes according to age group. Short term cessation programmes, such as those currently offered in schools, had limited appeal for these participants. The adolescent girls suggested that longer term support and follow up would improve the success of these programmes. The authors found that peer and family support were often lacking for girls who wanted to stop smoking. Nurses need to work with the families and peers to ensure that necessary supports for stopping smoking are in place.

Finally, the study identified withdrawal symptoms as a real barrier to stopping smoking; the girls, however, did not view the use of cessation aids, such as the nicotine patch and chewing gum, as something they would use. Nurses who work with adolescents may be able to increase the appeal of these aids and provide the support and supervision necessary for girls to use them effectively. Because the cost of these drugs could be an additional barrier to their use, nurses can play a part in finding creative ways to finance this type of medical assistance. Overall, the results of this study suggest that a combination of supports are necessary to assist adolescent girls in their efforts to stop smoking.

Marjorie MacDonald, RN, $\mathrm{PhD}$ Assistant Professor, School of Nursing University of Victoria

Victoria, British Columbia, Canada 